\title{
Improved inductance calculation in variable power inductors by adjustment of the reluctance model through magnetic path analysis
}

\author{
Sarah Saeed ${ }^{1}$, Jorge Garcia ${ }^{1}$, Marina S. Perdigão ${ }^{2,3}$, Valter S. Costa ${ }^{2,4}$, Bruno Baptista ${ }^{5}$ and André M. S. Mendes ${ }^{2,4}$ \\ ${ }^{1}$ Electrical Engineering Department, University of Oviedo, Spain, \\ ${ }^{2}$ Instituto de Telecomunicações, Coimbra, Portugal, \\ ${ }^{3}$ Coimbra Polytechnic - ISEC, Coimbra, Portugal, \\ ${ }^{4}$ Universidade de Coimbra, Coimbra, Portugal, \\ ${ }^{5}$ WEGeuro - Indústria Eléctrica, S.A. 4470-605 Maia, Portugal. \\ email: saeedsarah@uniovi.es
}

\begin{abstract}
This paper presents a study to improve the inductance calculation of magnetic elements for power converters, particularly important in the case of applications using variable inductors. Several factors have been taken into consideration, such as: the permeability definition under saturation, the temperature effect on the magnetic material behavior, and the distribution of saturated regions in the magnetic core which results in an accurate estimation of reluctance paths. Further depth is given to the study by using Finite Element Analysis to simulate a variable inductor and analyze the effect of the latter mentioned factors. The studied analysis is then compared with experimental results to prove the validity of the proposed improvements.
\end{abstract}

Keywords-FEA modeling, variable inductor, reluctance circuit, permeability, controlled saturation.

\section{INTRODUCTION}

In power electronic converters, the design of the power inductor plays an essential role in the performance of the whole system, as it can significantly impact the size and the power density of the converter. Therefore, it is necessary to develop accurate models of this device as well as the study of the limitations of its design such as material saturation, core losses, etc.

In principle, the modeling methods are confined to either analytical or numerical methods. Specific to variable magnetic devices, modeling strategies are mainly: Finite Element Analysis (FEA) [1][2], gyrator-capacitor model [3], and reluctance equivalent circuit [4].

FEA models are based on numerical analysis, and are considered very powerful to analyze the behavior of magnetic components especially under nonlinear operation. However, they have some drawbacks such as the sensitivity of the solution to geometrical dimensions and material properties, also the amount of data that needs to be post-processed for 3D simulations [5], and moreover, it is not straightforward to move between the magnetic solution and the corresponding electrical circuit model.

The work has been partially supported by the Spanish Government, under the research grant ENE2016-77919, “Conciliator" Project (Innovation Development and Research Office-MEC). Also, the work has been partially supported by the government of the Principality of Asturias, grant no. FC-GRUPINIDI/2018/000241, and under "Severo Ochoa" program of predoctoral grants for training in research and university teaching, grant number BP16-133.
Therefore, the methodology followed in this paper is to use the FEA model during the design stage of the magnetic device to acquire the necessary knowledge about the magnetic core behavior under saturation, such as the permeability definition, the flux distribution, and the temperature effect. The effect of those factors can thus be aggregated to the appropriate analytical circuit model. This allows the use of analytical-based models without compromising the accuracy acquired by the FEA-based ones.

In this study, the reluctance equivalent circuit is employed to model the magnetic device. Particularly, the proposed model is applied to study the variable inductor. In general, variable magnetic elements allow for additional degrees of freedom in the design and control of PECs. This is distinctly useful in resonant converters, where the usual frequency control has some drawbacks due to Electro-Magnetic Interference (EMI) issues, synchronization, variable sampling time, etc., especially for large range of variation. If variable magnetics are used, the same control margins can be obtained at a constant switching frequency, therefore allowing for an optimization of the EMI filters, and sampling procedures. In other applications such as the Dual Active Bridge (DAB) converter, in addition to adding a new degree of freedom to the control, the inclusion of variable magnetics can increase operation parameters such as the soft switching margins, etc. [6][7][8].

One of the issues of modeling the variable inductance is the inaccuracy of the estimation of the magnitude of inductance variation. This issue arises due to the ill-defined material properties under saturation conditions [9]. The aim of this paper is to set the accurate definitions for the magnetic material properties under saturation operation of the variable inductor magnetic core, such as the permeability, the $\mathrm{B}(\mathrm{H})$ curve, and the distribution of saturated regions in the core, which impacts the reluctance path lengths. Therefore, a model is proposed to calculate the accurate inductance value, as well as the magnitude of the inductance variation under different saturation conditions.

In this context, section II explains the reluctance equivalent model corresponding to the studied variable inductor. In section III, the modeling of the device using FEA is presented. Based on the analysis in the latter section, section IV discusses the proposed analytical reluctance model. And finally, the conclusions of the work are summarized. 


\section{Reluctance EQUivalent CIRCUIT}

As mentioned before, the reluctance equivalent circuit is used to model the magnetic device under study. Specific to this study, the variable inductor structure used is the double E-core structure [10] depicted in Fig. 1. Due to the main winding $\left(N_{c}\right)$, an AC flux $\left(\phi_{C}\right)$ circulates through the center arm and splits to the outer arms. Applying a relatively small DC current $\left(I_{b}\right)$ to the bias control windings $\left(N_{b}\right)$, a DC flux $\left(\phi_{R}\right.$ or $\left.\phi_{L}\right)$ is produced which circulates mainly through the outer (ungapped) circumference of the core [10]. This flux can bias the operation of the magnetic material towards nonlinear region, causing the inductance seen from the main winding terminals to vary [4][11].

The reluctance equivalent circuit is composed of three main elements [4]; a) a constant reluctance which models any non-ferromagnetic material such as air gap, b) a variable reluctance which models the behavior of the ferromagnetic material, and c) a winding model which represents the magnetomotive ( $\mathrm{mmf}$ ) or Ampere-turn of the winding. Fig. 2a illustrates the lengths of different reluctance paths and Fig. $2 b$ shows the equivalent reluctance circuit model of this magnetic core. The latter circuit can be simplified to the reluctance circuit shown in Fig. 3. Accordingly, the inductance can be calculated based on the reluctances as shown by (1).

$$
L=\frac{N_{c}{ }^{2}}{\mathcal{R}_{C}+\mathcal{R}_{\text {gap }}+\mathcal{R}_{L} / / \mathcal{R}_{R}}
$$

where $\mathcal{R}_{C}, \mathcal{R}_{L}$, and $\mathcal{R}_{R}$ are the reluctances of the center, left and right arms, respectively. The reluctance of a magnetic path is calculated by (2).

$$
\mathcal{R}_{i}=\frac{l_{i}}{\mu_{i} \cdot A_{i}}
$$

where $l_{i}$ is the length of the path $i, \mu_{i}$ is the permeability of the magnetic material, and $A_{i}$ is the cross sectional area. The reluctance, thus, is a function of the permeability, which in turn depends on the $\mathrm{B}(\mathrm{H})$ curve of the magnetic material. Operating at the knee or the saturation regions of the $\mathrm{B}(\mathrm{H})$ curve, the magnetic material will have completely nonlinear behavior, so the definition of the permeability will change as well. Moreover, under different operation points, the $\mathrm{B}(\mathrm{H})$ curve of the material changes as a function of the operation temperature of the core. Therefore, the calculation of the permeability, and thus the inductance, becomes a nontrivial task. To attain a deeper understanding of the behavior of the magnetic core under saturation, FEA methodology is used to construct, simulate, and analyze the magnetic device under different operation conditions.

\section{MOdel of VARIABLE INDUCTOR USING FEA}

The software package used for FEA simulations is Altair Flux $^{\mathrm{TM}}$ by Cedrat [12]. Briefly, the FEA simulation is developed in three main steps; first, the construction of the 3D core geometry and the mesh generation. Second, adjusting the physical properties which include defining the materials of the magnetic core and the coils, constructing coil conductors, and assigning different volumes regions. And thirdly, solving the constructed magnetic device under certain scenarios of parameters. The influence of any parameter in a simulation can be explored and visualized through multidimensional curves and animations of color shades or arrows.

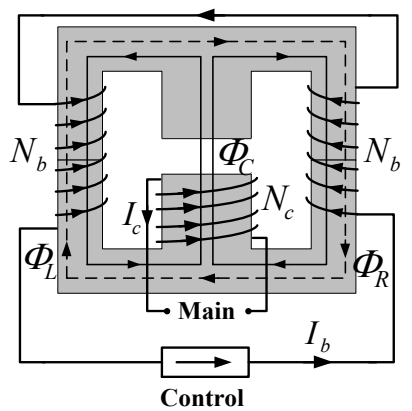

Fig. 1. Variable inductor based on double E-core [10].

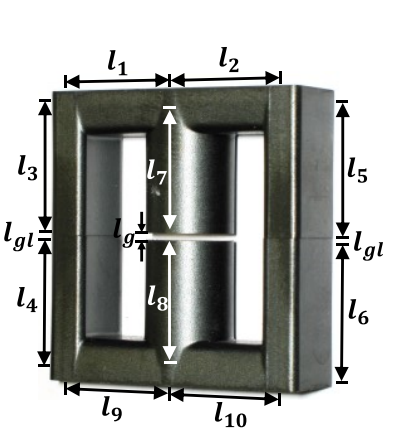

(a)

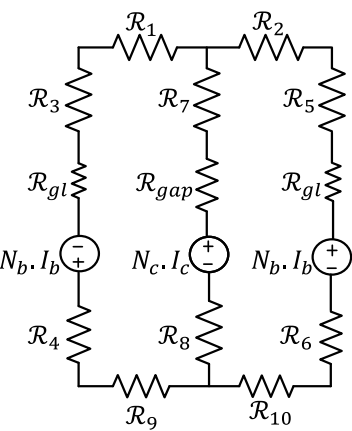

(b)
Fig. 2. Reluctance circuit - (a) Lengths of reluctance paths, and (b) the corresponding circuit model.

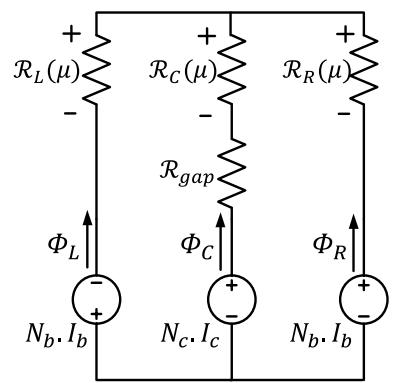

Fig. 3. Reluctance equivalent circuit of the variable inductor in Fig.1.

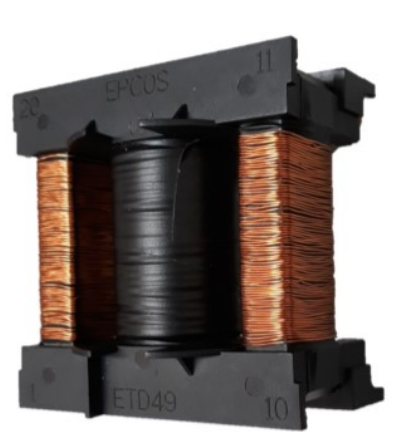

(a)

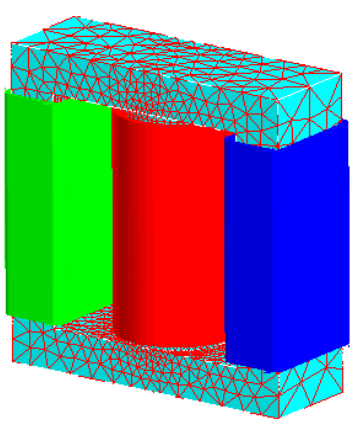

(b)
Fig. 4. Variable inductor -a) Prototype based on double Ecore, and b) constructed 3D model in Flux ${ }^{\mathrm{TM}}$ software illustrating the meshed magnetic core and the three windings.

\section{A. Construction}

The variable inductor was constructed in Flux ${ }^{\mathrm{TM}} 3 \mathrm{D}$, using the dimensions measured by a caliper, to accurately resemble the constructed prototype as it can be observed in Fig. 4. The specifications of the designed variable inductor are stated in Table I. 
TABLE I. SPECIFICATIONS OF VARIABLE INDUCTOR PROTOTYPE

\begin{tabular}{|c|c|}
\hline Core Size & ETD49/25/16 \\
\hline Magnetic Material & Ferrite N87 \\
\hline $\boldsymbol{\Delta L}$ & $147 \mathrm{uH}$ to $53 \mathrm{uH}$ \\
\hline $\boldsymbol{N}_{\boldsymbol{c}}$ & 23 turns \\
\hline $\boldsymbol{N}_{\boldsymbol{b}}$ & 72 turns \\
\hline $\boldsymbol{l}_{\text {gap }}$ & $1 \mathrm{~mm}$ \\
\hline
\end{tabular}

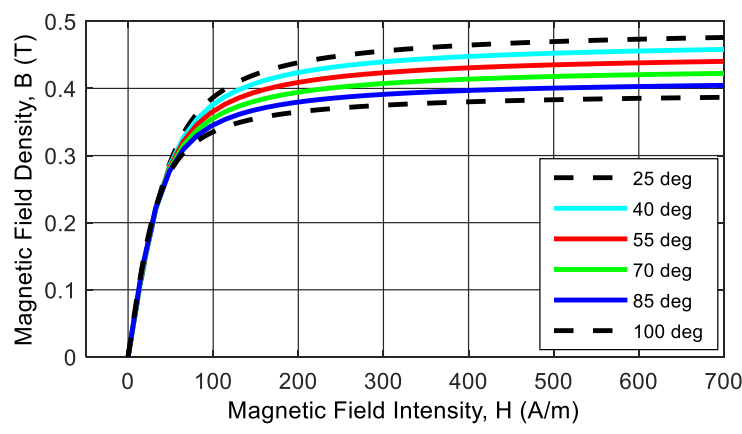

Fig. 5. $\mathrm{B}(\mathrm{H})$ curve of $\mathrm{N} 87$ magnetic material.

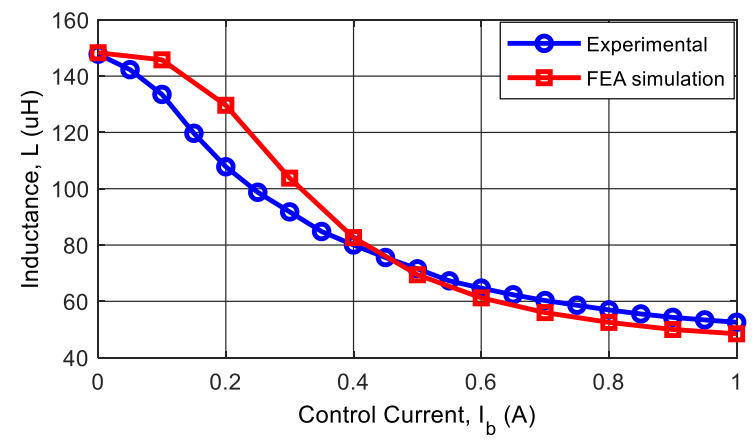

Fig. 6. Inductance as a function of bias control current - FEA computation compared to the small-signal experimental measurements.
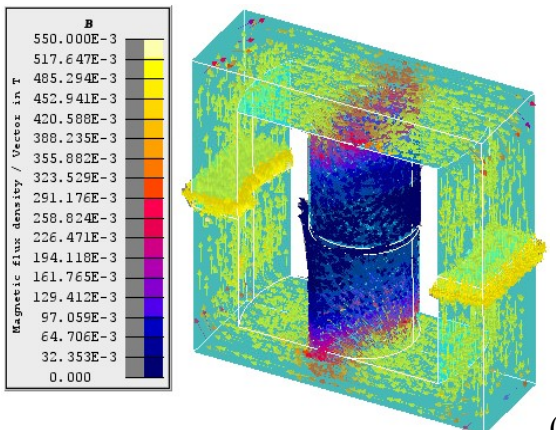

(a)
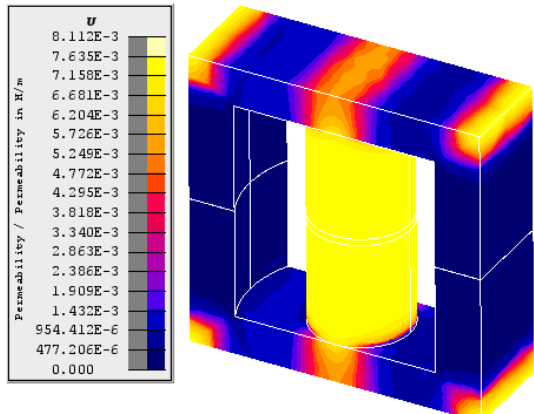

(b)

Fig. 7. FEA simulation results - a) Distribution of magnetic flux density, and b) distribution of permeability in the core.
The $\mathrm{N} 87$ magnetic material $\mathrm{B}(\mathrm{H})$ curves at $25^{\circ} \mathrm{C}$ and $100^{\circ} \mathrm{C}$ were extracted from the datasheet. Then, by using "Interpolation Over a Grid" (meshgrid command in Matlab), the $\mathrm{B}(\mathrm{H})$ curves at different temperatures were obtained and introduced to the FEA model, as shown in Fig. 5. Linear interpolation was assumed based on the fact that the permeability and the core loss parameters, provided by the magnetic core manufacturer [13], show linear behavior as a function of the temperature for the range between $25^{\circ} \mathrm{C}$ and $100^{\circ} \mathrm{C}$. The windings' copper material properties were added to the FEA model as well. And finally, the materials were assigned to the corresponding volume regions.

\section{B. Inductance Calculation}

A scenario was configured to vary the DC current in the control windings from 0 to $1 \mathrm{~A}$ in steps of 0.1 , in order to calculate the inductance value under different bias conditions. In Flux ${ }^{\mathrm{TM}}$ software, one of the ways to compute the inductance is by using a sensor based on the theoretical definition expressed by (3).

$$
L=\frac{N_{C} \cdot \Phi_{C}}{I_{C}}
$$

where $I_{c}$ is the AC current in the inductor main winding, and $\phi_{C}$ is the AC flux linking this winding. The computed inductance was compared to the experimental value obtained from smallsignal measurements using an impedance analyzer model BK Precision 889A Bench LCR/ESR Meter. During small-signal measurements, the main winding current is relatively small, less than $0.1 \mathrm{~A}$, therefore, the same value of current was set in the FEA model to assure accuracy when comparing results. Fig. 6 shows the inductance as a function of the DC bias current. The error between the modeled and measured values is observed to be less than $20 \%$, which is an acceptable value.

\section{Analysis of Saturation of Magnetic Core}

Using the graphical visualization of the results in the Flux $^{\mathrm{TM}}$ 3D software, it is more apprehensive to learn about complex quantities such as magnetic flux density, permeability of the magnetic core, etc. and their distribution across the core. Fig. 7a illustrates the 3D core model with the distribution of magnetic flux density. Different arrows represent the orientation of magnetic flux density, and their colors correspond to the value of magnetic flux density. Similarly, Fig. 7b illustrates the 3D core model with the distribution of permeability. Color shading corresponds to the value of magnetic permeability in each zone [2].

\section{Proposed Vi Analytical Reluctance Model}

As it was clarified by the previous sections, the variable inductor device is distinguished by its the capability to vary the inductance value. The range of the variation of the inductance depends on the design of the device, however, the design procedure does not guarantee the desired maximum and minimum inductance values. This process is usually iterative and is one of the issues of modeling the variable inductor. The issue arises due to ill-defined material properties under saturation conditions. In this section, a reluctance circuit model is proposed which provides an accurate estimation of the inductance as a function of the bias control current. The model sets the accurate definitions for the magnetic material properties such as the permeability, the $\mathrm{B}(\mathrm{H})$ curve, and the distribution of saturated regions in the core, which impacts the reluctance path lengths. 


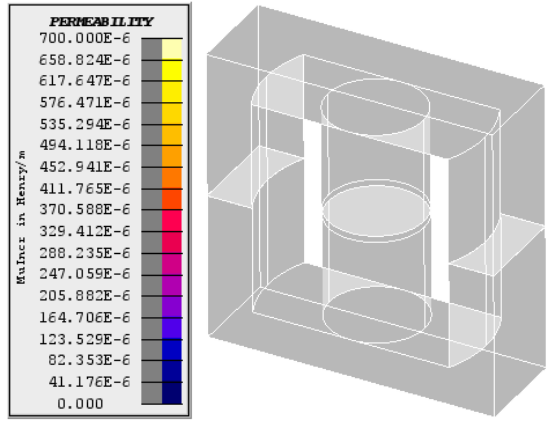

(a)

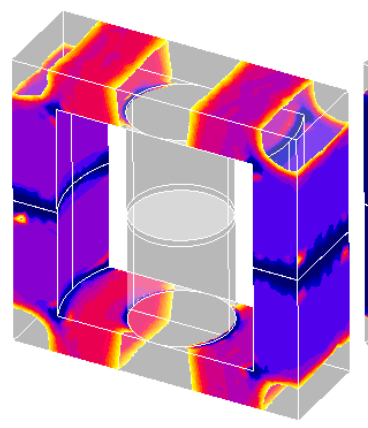

(b)

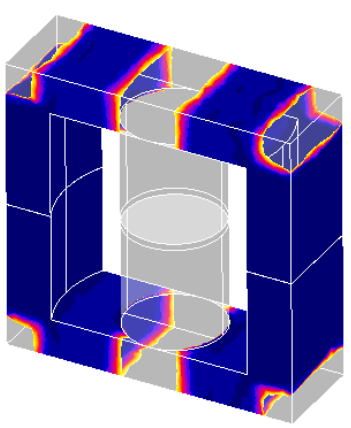

(c)

Fig. 9. FEA simulation results - Differential permeability across the magnetic core at DC bias current of: a) $0 \mathrm{~A}$, b) $0.3 \mathrm{~A}$, and c) $1 \mathrm{~A}$.

\section{A. Magnetic Permeability Definition}

The permeability differs in definition corresponding to the relation between the magnetic flux density (B) and the magnetic field intensity $(\mathrm{H})$ for a certain magnetic material [14], as explained by (4).

$$
\mu=B / H
$$

However, the permeability has different interpretations depending on the conditions of operation and measurement. A summary of different permeability definitions is provided in [14]. When an alternating magnetic field (AC) is superimposed on a static magnetic field (DC), a minor hysteresis loop is produced, resulting in the incremental permeability, $\mu_{\Delta}$, defined by (5).

$$
\mu_{\Delta}=\Delta B / \Delta H
$$

If the area of the minor loop is relatively small, it reduces to a straight line and therefore, the incremental permeability can be approximated to the differential permeability [15], $\mu_{d}$, defined by (6).

$$
\mu_{d}=d B / d H
$$

The differential permeability, in this context, has been reported to give the most accurate definition of the permeability in nonlinear and multi-winding systems [9] [16]. Therefore, it will be used throughout this study.

Specific to the N87 ferromagnetic material, Fig. 8 compares the permeability, calculated from (4), to the differential permeability, calculated from (6), as a function of magnetic field intensity.

Also, Fig. 9 shows the differential permeability values graphically represented on the 3D core model in an evolution from no saturation, Fig. 9a, through partial saturation, Fig. 9b, and until maximum saturation, Fig. 9c. As it can be seen, the permeability values are nonlinearly distributed across the core and shows an evolution of length of saturated paths in the core. Under maximum saturation condition, some parts of the magnetic core, specifically the corner points, are not utilized or in other words unsaturated demonstrating much higher permeability values. Therefore, this issue must be taken into consideration when applying the reluctance circuit to model the magnetic core under saturation. The lengths of different reluctance paths in the magnetic core should be modified according to the saturation condition to correspond to the actual saturated portions of the core [9].

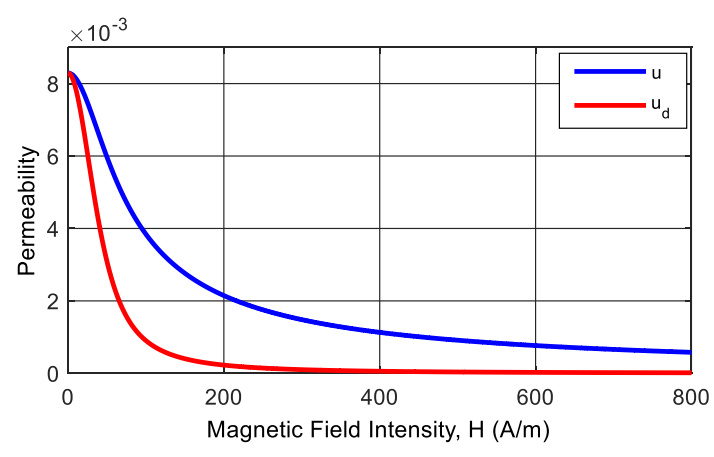

Fig. 8. Different permeability definitions for N87 ferromagnetic material.

\section{B. Modification of Reluctance Path Lengths}

As it was explained in the previous section, under saturation of the magnetic core, the lengths of different reluctance paths are altered. Specific to the double E-core variable inductor, the saturation takes place mainly in the outer circumference of the core, as explained in section II. Therefore, the lengths of the paths forming this outer frame of the core are modified. As defined by Fig. $2 \mathrm{a}, l_{l}, l_{3}, l_{4}$, and $l_{9}$ represent the left magnetic path, while $l_{2}, l_{5}, l_{6}$, and $l_{10}$ represent the right magnetic path. Due to symmetry, $l_{1}$ and $l_{3}$ are calculated and applied to the corresponding lengths of the core.

Equations (7) and (8) state the calculation of the modified lengths of those reluctance paths under saturation, as clarified by Fig. 10. Those modified lengths are calculated as a function of the magnetic core dimensions defined by Fig. 11.

$$
\begin{gathered}
\left.l_{1}\right|_{\text {sat }}=\frac{w_{3}}{16}+\frac{w_{2}-w_{3}}{2}+\frac{w_{1}-w_{2}}{8} \\
\left.l_{3}\right|_{\text {sat }}=a_{2}+\frac{a_{1}-a_{2}}{4}
\end{gathered}
$$

The initial approach to obtain those equations are based on experimental observations during several tests carried out on E-core variable inductors and first presented in [9]. In this latter literature work, the equations were verified for the magnetic core sizes: ETD49, EFD25 and EF25, as well as for different values of air-gaps. Therefore, the equations are considered accurate for E-shaped variable inductor cores.

The modified reluctance path lengths are then substituted in (2) along with the value of the differential permeability, defined by (6), to calculate the reluctances of different magnetic paths. 


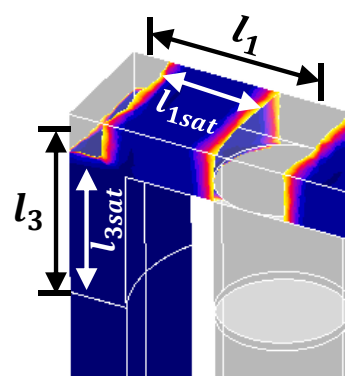

Fig. 10. Definition of modified reluctance paths in the proposed reluctance model.

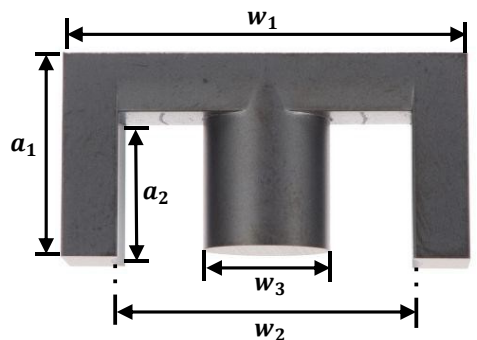

Fig. 11. Dimensions of the magnetic core.

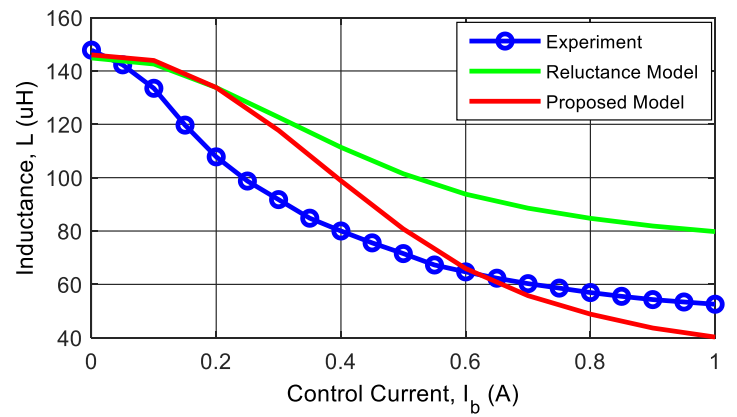

Fig. 12. Inductance calculation - Proposed analytical reluctance model versus original reluctance equivalent circuit.

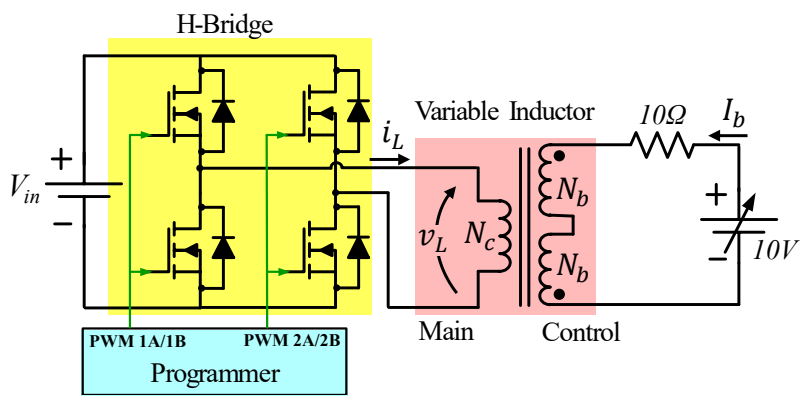

Fig. 13. Circuit diagram of the test platform.

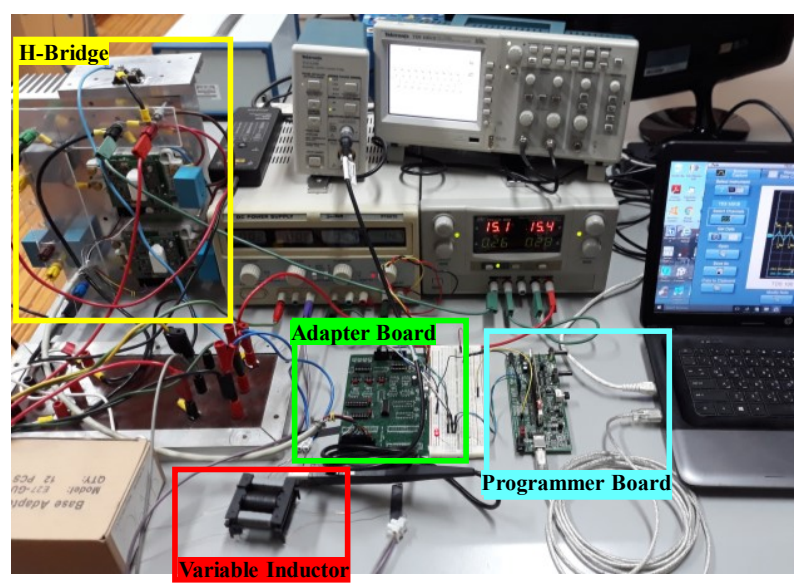

Fig. 14. Experimental test setup.
Consequently, to compute the inductance, the reluctance values are substituted in (1). Also, the fringing effect due to the air gap was considered [17], so the inductance value is corrected by a factor, $X_{f}$, which is calculated by (9).

$$
X_{f}=1+\frac{l_{g}}{\sqrt{A_{c}}} \cdot \ln \left(\frac{2 \cdot h_{w}}{l_{g}}\right)
$$

where $h_{w}$ is the total height of the main winding, and $A_{c}$ is the cross section area of the center arm. The fringing factor computed for the magnetic core under study is 1.3. And accordingly, the inductance corrected for fringing, $L^{\prime}$, is computed by (10).

$$
L^{\prime}=L \cdot X_{f}
$$

Fig. 12 illustrates the inductance as a function of the control current. The inductance value obtained from the proposed reluctance model gives more accuracy compared to the experimental measurements especially as the saturation current increases, where the original reluctance circuit model is seen to disperse from those values.

\section{Temperature Effect on $B(H)$ Curve and its Impact on Inductance}

From the above discussions, it can be concluded that the inductance value depends on the operation point of the magnetic material on the $\mathrm{B}(\mathrm{H})$ curve. The $\mathrm{B}(\mathrm{H})$ curve of the material can vary depending on the temperature of operation as well. There are no previous studies which considered the effect of temperature dependence of $\mathrm{B}(\mathrm{H})$ curve on the inductance calculation in variable inductors. Specific to those devices, the temperature of the core can have a significant effect on the range of variation of the inductance, which in the end impacts the design of the device. In this work, some experimental measurements are carried out to understand the temperature effect on the inductance variation range, and eventually include this effect in the full design process of the variable inductor.

Fig. 13 shows a circuit diagram of the test platform used for the experiments. An H-bridge is constructed to apply an AC square waveform excitation voltage to the inductor main winding. A DC current is applied to the inductor control windings which is obtained by a DC voltage supply connected in series with a resistance to have a maximum of $1 \mathrm{~A}$. The test setup is illustrated in Fig. 14, and the design specifications are listed in Table II.

TABLE II. SPECIFICATIONS OF TEST SETUP

\begin{tabular}{|c|c|}
\hline \multicolumn{2}{|c|}{ H-bridge } \\
\hline IGBT Module & $\begin{array}{c}\text { Semikron } \\
\text { SEMiX202GB066HDS }\end{array}$ \\
\hline Adapter board & Board 2S SKYPER 32 PRO \\
\hline Driver Core & SKYPER 32 PRO \\
\hline \multicolumn{2}{|c|}{ Programmer } \\
\hline DSP Board & Texas Instruments \\
\hline \multicolumn{2}{|c|}{ TMS320F28335 } \\
\hline Core Size Inductor \\
\hline Magnetic Material & ETD49/25/16 \\
\hline Inductance variation range, $\boldsymbol{\Delta L}$ & $147 \mathrm{uH}$ to 53uH \\
\hline \multicolumn{2}{|c|}{ Operation Conditions } \\
\hline Input voltage, $\boldsymbol{V}_{\text {in }}$ & $0-50 \mathrm{~V}$ \\
\hline Switching frequency, $\boldsymbol{f}$ & $20 \mathrm{kHz}$ \\
\hline Inductor peak current & $10 \mathrm{~A}$ \\
\hline Temperature & $25^{\circ} \mathrm{C}-70^{\circ} \mathrm{C}$ \\
\hline
\end{tabular}


Three tests have been carried out at three different DClink input voltages, $\mathrm{V}_{\mathrm{in}}, 10 \mathrm{~V}, 30 \mathrm{~V}$, and $50 \mathrm{~V}$. At each operation condition, the inductance value is varied from maximum to minimum values by means of varying the control current from 0 to $1 \mathrm{~A}$. Fig. 15 shows the waveform of the main winding current, $i_{L}$, for the three operation conditions at $1 \mathrm{~A}$ control current.

The inductance value is calculated using the RMS values of the first harmonic component of the voltage and current measurements, so the resulting inductance is calculated by (11).

$$
L=\frac{X_{L}}{\omega}=\frac{V_{L}}{I_{L}} \cdot \frac{\pi / 4}{2 \pi \cdot f}
$$

where $V_{L}$ is the RMS value of the voltage measured across the main winding of the variable inductor, and $I_{L}$ is the RMS value of current flowing through this winding. It is worth to note that using the fundamental components simplifies the calculation of the inductance without compromising the accuracy of the measured value. This conclusion was based on comparing the results obtained by using this method to those obtained by using instantaneous measured waveforms applied to System Identification Tool from Matlab. The inductance values calculated showed a very close agreement which justifies the simplification. Fig. 16a illustrates the inductance curves as a function of the control current for each power level. The inductance variation range is calculated by (12).

$$
\Delta L=\left(L_{\max }\right)_{I_{b}=0}-\left(L_{\min }\right)_{I_{b}=1}
$$

It can be observed that as the power level increases, from $10 \mathrm{~V}_{\text {in }}$ to $50 \mathrm{~V}_{\text {in }}$, the inductance variation range is reduced from $92 \mathrm{uH}(143-51 \mathrm{uH})$ to $69 \mathrm{uH}(143-74 \mathrm{uH})$, which is around $16 \%$ reduction.

The temperature was recorded during each test by using FLIR thermal camera. Fig. 16b illustrates the temperature curves as a function of the control current for each power level. The temperature gradient is calculated by (13).

$$
\Delta T=\left(T_{\max }\right)_{I_{b}=1}-\left(T_{\min }\right)_{I_{b}=0}
$$

It can be observed that as the power level increases, from $10 \mathrm{~V}_{\text {in }}$ to $50 \mathrm{~V}_{\text {in }}$, the temperature gradient is increased from $11.6^{\circ} \mathrm{C}\left(38.6-27^{\circ} \mathrm{C}\right)$ to $28.2^{\circ} \mathrm{C}\left(65.9-37.7^{\circ} \mathrm{C}\right)$. The temperature gradient, $\Delta T$, is plotted against the corresponding inductance variation range, $\Delta L$, as shown in Fig. 17. It can be concluded that the inductance variation range is inversely proportional to the temperature gradient, as clarified by (14).

$$
\Delta L \alpha \frac{1}{\Delta T}
$$

It has been observed from the experiments that there is a cross coupling between the temperature effect and the value of the inductor current flowing through the main winding. Therefore, the variation range of the inductance, is not only affected by the temperature of operation of the core, but also by the $m m f$ of the main winding. A knowledge of the relationship between the inductance value, temperature of operation, and the inductor current is therefore essential to estimate an accurate value of the inductance under saturation conditions particularly at higher power levels of inductor operation.

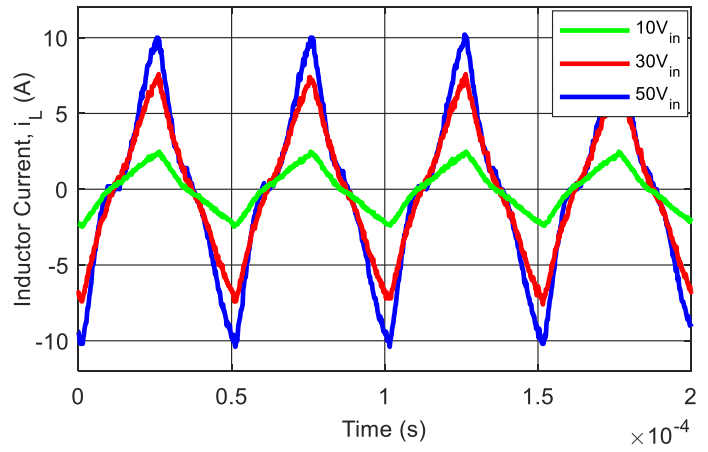

Fig. 15. Inductor current waveform at $1 \mathrm{~A}$ control current for three different operation conditions at DC-Link input voltage of $10 \mathrm{~V}, 30 \mathrm{~V}$, and $50 \mathrm{~V}$.

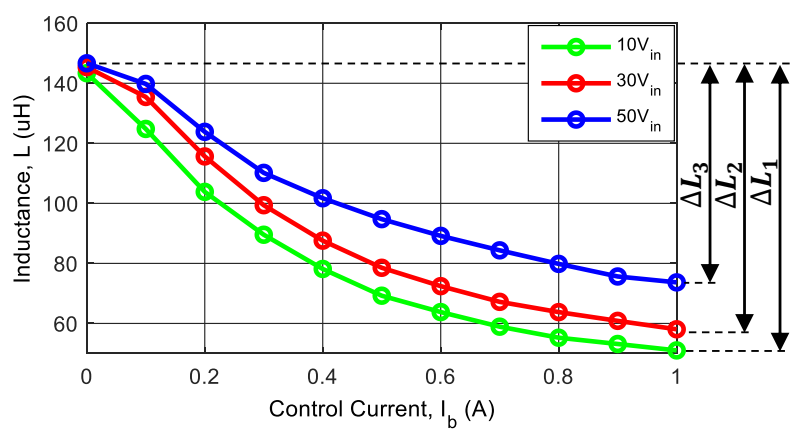

(a)

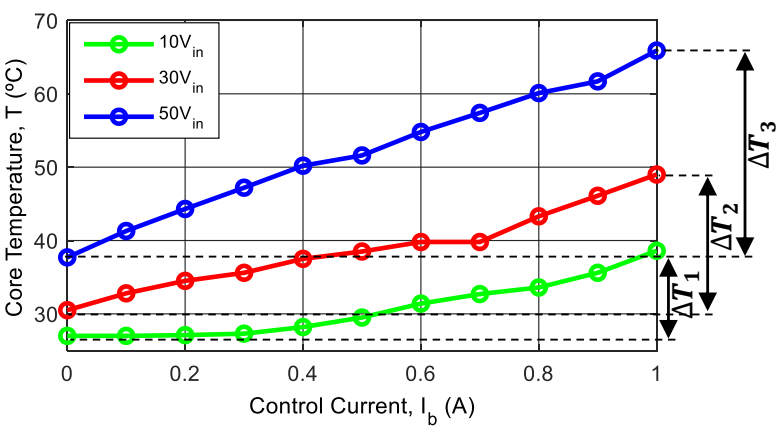

(b)

Fig. 16. Temperature effect on inductance value - a) measured inductance, and b) corresponding temperature as a function of bias current.

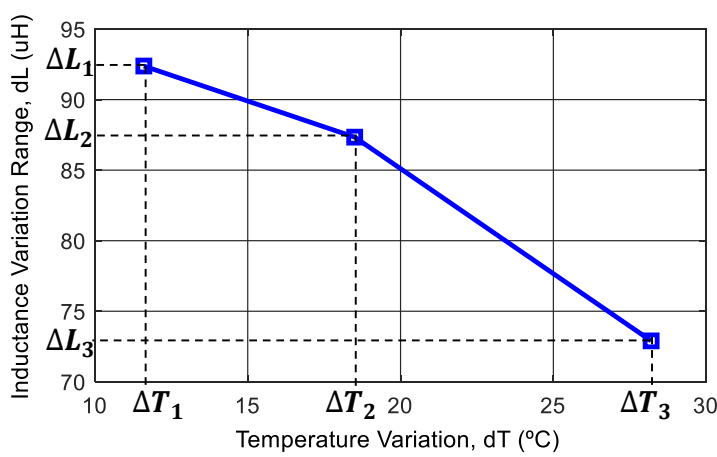

Fig. 17. Inductance variation range as a function of temperature gradient. 


\section{CONCLUSIONS}

The paper presented a study of the inductance calculation in variable inductor. A modified analytical reluctance model has been proposed, which gives better accuracy to the inductance calculation especially under saturation of the magnetic core. The contributions of the proposed model compared to the conventional reluctance equivalent circuit consist in three main points. First, the permeability is redefined to use the differential permeability for describing the nonlinear behavior of the magnetic material. Second, through simulations of the device using finite element analysis, more insight was given to the permeability distribution in the magnetic core under saturation. Based on these simulations, the reluctance path lengths were modified to exclude unutilized partitions of the core. Thirdly, the temperature dependence of the magnetic material has been explored through experimental measurements to study its effect on inductance variation range.

The proposed analytical reluctance model, therefore, has a compared accuracy to that of the FEA-based model. The adjustments proposed in this paper allow to bridge the gap between the accuracy provided by the FEA simulations and that of analytical solutions in describing the nonlinear behavior of the variable inductor.

\section{REFERENCES}

[1] Y. Bi and D. C. Jiles, "Finite element modeling of an electrically variable inductor," in IEEE Transactions on Magnetics, vol. 35, no. 5, pp. 3517-3519, Sept. 1999.

[2] M. S. Perdigão, S. F. Ferreira, M. Martins, A. S. Mendes and J. M. Alonso, "Finite element analysis of a variable inductor for an RSCC based LED lamp driver," 2015 IEEE Industry Applications Society Annual Meeting, Addison, TX, 2015, pp. 1-8.

[3] D. C. Hamill, "Gyrator-capacitor modeling: a better way of understanding magnetic components," Proceedings of 1994 IEEE Applied Power Electronics Conference and Exposition - ASPEC'94, Orlando, FL, USA, 1994, pp. 326-332 vol.1.

[4] J. M. Alonso, G. Martínez, M. Perdigão, M. Cosetin and R. N. do Prado, "Modeling magnetic devices using SPICE: Application to variable inductors," 2016 IEEE Applied Power Electronics Conference and Exposition (APEC), Long Beach, CA, 2016, pp. 1115-1122.

[5] R. Prieto, R. Asensi, C. Fernandez, J. A. Oliver and J. A. Cobos, "Bridging the Gap Between FEA Field Solution and the Magnetic Component Model," in IEEE Transactions on Power Electronics, vol. 22, no. 3, pp. 943-951, May 2007.
[6] H. Fan and H. Li, "High-Frequency Transformer Isolated Bidirectional DC-DC Converter Modules with High Efficiency Over Wide Load Range for $20 \mathrm{kVA}$ Solid-State Transformer," in IEEE Transactions on Power Electronics, vol. 26, no. 12, pp. 3599-3608, Dec. 2011.

[7] S. Saeed and J. Garcia, "Extended Operational Range of Dual-ActiveBridge Converters by using Variable Magnetic Devices," 2019 IEEE Applied Power Electronics Conference and Exposition (APEC), Anaheim, CA, USA, 2019, pp. 1629-1634.

[8] Burgio, A.; Menniti, D.; Motta, M.; Pinnarelli, A.; Sorrentino, N.; Vizza, P., "A laboratory model of a dual active bridge DC-DC converter for a smart user network," in Environment and Electrical Engineering (EEEIC), 2015 IEEE 15th International Conference on, vol., no., pp.997-1002, 10-13 June 2015.

[9] Ferreira, Samuel Filipe Soares. Electromagnetic study of a variable inductor controlled by a DC current. MS thesis. University of Coimbra, 2016.

[10] D. Medini and S. Ben-Yaakov, "A current-controlled variable inductor for high frequency resonant power circuits," in Proc. IEEE APEC, 1994, pp. 219-225.

[11] S. Saeed, J. García and R. Georgious, "Modeling of variable magnetic elements including hysteresis and Eddy current losses," 2018 IEEE Applied Power Electronics Conference and Exposition (APEC), San Antonio, TX, 2018, pp. 1750-1755.

[12] Altair Flux ${ }^{\mathrm{TM}} \quad$ [Online]. Available: https://altairhyperworks.com/product/flux\#

[13] Ferrites and accessories, TDK [Online]. Available: https://www.tdkelectronics.tdk.com/download/528882/71e02c7b9384de1331b3f625ce 4b2123/pdf-n87.pdf

[14] A. Van den Bossche and V. C. Valchev, Inductors and Transformers for Power Electronics, 2005.

[15] W. J. Croisant, C. A. Feickert and M. K. McInerney, "A differential magnetic permeability model for pulsed magnetic field calculations," in IEEE Transactions on Magnetics, vol. 32, no. 5, pp. 4326-4328, Sept. 1996.

[16] M. S. Perdigão, M. F. Menke, Á. R. Seidel, R. A. Pinto and J. M. Alonso, "A Review on Variable Inductors and Variable Transformers: Applications to Lighting Drivers," in IEEE Transactions on Industry Applications, vol. 52, no. 1, pp. 531-547, Jan.-Feb. 2016

[17] A. Van den Bossche, V. Valchev and T. Filchev, "Improved approximation for fringing permeances in gapped inductors," Conference Record of the 2002 IEEE Industry Applications Conference. 37th IAS Annual Meeting (Cat. No.02CH37344), Pittsburgh, PA, USA, 2002, pp. 932-938 vol.2. 